\title{
ON THE REGULARITY OF THE RIEMANN FUNCTION FOR HYPERBOLIC EQUATIONS
}

\author{
BY
}

\author{
WILLIAM L. GOODHUE( ${ }^{(1)}$
}

ABSTRACT. In an earlier paper, A. Friedman demonstrated that the Riemann function for a strictly hyperbolic system with Gevrey coefficients was locally Gevrey of some higher order except along the bicharacteristics. By representing the Riemann function in terms of a progressing wave expansion, this result is extended beyond caustics.

Introduction. In a 1962 paper A. Friedman [2] showed for a linear, strictly hyperbolic system of partial differential equations that off the bicharacteristics the Riemann function is locally Gevrey if the coefficient matrices are locally Gevrey of some lower order. It had previously been demonstrated by R. Courant and P. Lax [1] that the Riemann function was locally $C^{\infty}$ off the bicharacteristics.

In 1959 D. Ludwig [5] sketched a proof of the extension of the Courant and Lax result beyond the caustics, although it remained for J. Ralston in an unpublished paper to give a complete proof based upon Ludwig's ideas. Using Ralston's technique it will be shown that it is possible to extend Friedman's result beyond caustics, except for the case of analytic coefficients, where the local result seems to be the best possible one.

The author wishes to express his thanks to D. Ludwig and J. Ralston for their help and to P. D. Lax who suggested the original problem and who has been a constant source of encouragement and enlightenment.

I. Regularity of the Riemann function. The Riemann function for the hyperbolic system Cauchy problem

$$
\frac{\partial}{\partial t} u(x, t)=\sum_{j=1}^{k} A^{j}(x, t) \frac{\partial}{\partial x_{j}} u(x, t)+B(x, t) u(x, t), \quad u(x, 0)=f(x)
$$

where $u$ is an $n$-vector in $x=\left(x_{1}, \cdots, x_{k}\right)$ and $t$, and the $A^{j}(x, t), B(x, t)$ are smooth $n \times n$ matrices, is a distribution valued matrix $R(y, s ; x, t)$, which depends on the pole $(y, s)$ and satisfies the Cauchy problem

Received by the editors December 27, 1971 .

AMS (MOS) subject classifications (1970). Primary 35L45, 35D10.

Key words and phrases. Riemann function, strictly hyperbolic systems, progressing wave expansion, Gevrey class.

(1) This work was derived from the author's doctoral dissertation, written under Professor Peter D. Lax (New York University, 1971). 


$$
\left[\frac{\partial}{\partial t}-\sum_{j=1}^{k} A^{j}(x, t) \frac{\partial}{\partial x_{j}}-B(x, t)\right] R(y, s ; x, t)=0, \quad t>s,
$$

$$
\begin{aligned}
& R(y, s ; x, s)=\delta(y-x) I, \\
& R(v, s ; x, t)=0, \quad t<s,
\end{aligned}
$$

$I$ being the $n \times n$ identity matrix. We require that the system be strictly hyperbolic; i.e., the characteristic form

$$
G(x, t, \xi)=\sum_{j=1}^{k} \xi_{j} A^{j}(x, t)
$$

has $n$ distinct eigenvalues $\left\{\tau^{\lambda}(x, t, \xi)\right\}_{\lambda=1}^{n}$ for each $k$-vector $\xi \neq 0$. The level surfaces of the functions $\phi^{\lambda}(x, t)$ which satisfy the first order differential equation

$$
\phi_{t}^{\lambda}(x, t)=\tau^{\lambda}\left(x, t, \operatorname{grad}_{x} \phi^{\lambda}(x, t)\right), \quad \phi^{\lambda}(x, 0)=\phi_{0}^{\lambda}(x)
$$

are called characteristic surfaces, and the assumption of strict hyperbolicity guarantees that the progressing wave expansions span the solution space of (1.1), modulo a smooth function [5]. These surfaces in turn are generated by the bicharacteristic rays $\left\{x_{i}^{\lambda}(t)\right\}_{i=1}^{k}$ which satisfy the Hamiltonian system

$$
\frac{d}{d t} x_{i}^{\lambda}(t)=\tau_{\xi_{i}}^{\lambda}(x, t, \xi), \quad \frac{d}{d t} \xi_{i}^{\lambda}(t)=-\tau_{x_{i}}^{\lambda}(x, t, \xi) .
$$

Once the Riemann function is determined, the solution to the Cauchy problem (1.1) is given by

$$
u(x, t)=\int R(y, 0 ; x, t) f(y) d y
$$

for any function $f(y) \in\left[L_{2}\left(R^{k}\right)\right]^{n}$.

P. D. Lax [3] and D. Ludwig [5] have shown that the Riemann function for (1.1) may be represented by the following progressing wave expansion

$$
\begin{aligned}
& R(y, s ; x, t)=\int_{|\omega|=1} R(y, s ; x, t, \omega) d \omega \\
& \quad=\int_{|\omega|} \sum_{j=0}^{N} \sum_{\lambda=1}^{n} f_{j}\left(\phi^{\lambda}(y, s ; x, t, \omega)\right) a_{j, \lambda}(y, s ; x, t, \omega) d \omega+R_{N}(y, s ; x, t),
\end{aligned}
$$

where $R(y, s ; x, t, \omega)$ satisfies the equation (1.2) with Cauchy data

$$
R(y, s ; x, t, \omega)=-\frac{1}{(2 \pi i)^{k}} \partial^{k} \log ((y-x) \cdot \omega)
$$

and $|\omega|=1$ is the unit ball in $R^{k}$. The $\phi^{\lambda}(y, s ; x, t, \omega)$ are characteristic surfaces with $\phi_{0}^{\lambda}=(y-x) \cdot \omega, f_{0}(z)=-1 /(2 \pi i)^{k} \cdot \partial^{k} \log (z), f_{j-1}(z)=d f_{j}(z) / d z$ for $j \geq 1$, and the functions $a_{j, \lambda}(y, s ; x, t, \omega)$ satisfy a sequence of transport equations. The crucial facts to keep in mind are that the $a_{j, \lambda}$ are $C^{\infty}$ functions, 
the distributions $f_{j}(\cdot)$ have their singular support on the set $\{0\}$, and for $N$ sufficiently large, the remainder term $R_{N}$ is arbitrarily smooth in all of its arguments. In fact, the result of Courant and Lax follows from the representation (1.7), as a basic theorem of differential equations guarantees the local existence of a nonsingular solution to (1.4).

To extend this result beyond the singularities of the characteristic surfaces, Ludwig suggested making use of the semigroup property for linear hyperbolic equations. Specifically, if

$$
U(t, s): u(x, s) \rightarrow u(x, t)
$$

represents the solution operator to (1.1), then for any time $\tau, 0<\tau<t$,

$$
u(x, t)=U(t, \tau) U(\tau, 0) f(x) .
$$

By constructing Riemann functions as above for the initial times 0 and $\tau$, he was able to conclude, using a geometric argument, that the kernel in (1.6) was $C^{\infty}$ except along the bicharacteristics coming from the pole at $(y, 0)$. The following proof, due to J. Ralston, is the analytic interpretation of this argument.

Theorem 1 . The Riemann function $R(y, s ; x, t)$ for the strictly byperbolic system (1.1) bas its singular support along the bicharacteristic through $(y, s)$.

Proof. Let $K>0$ and $R>0$ be chosen so that $\operatorname{supp}(f) \subset\{y:|y|<K\} \equiv B_{K}(0)$, and for some given $T_{1}>0, \operatorname{supp}\left(u\left(x, T_{1}\right)\right) \subset\{x:|x|<R\}=B_{R}(0)$. By a previous remark, for any given initial time $s, 0 \leq s \leq T_{0}\left(T_{0}\right.$ arbitrary) we can construct the kernel $R(y, s ; x, t)$ of the operator $U(t, s)$ using the technique of Lax and Ludwig for $s \leq t \leq s+T_{c}, y \in B_{K}(0), x \in B_{R}(0)$. If $0<T_{c} \leq T_{1}<2 T_{c}$, we choose $\rho(\tau) \in C_{0}^{\infty}$ so that $\int \rho(\tau) d \tau=1$ and $\operatorname{supp}(\rho) \subset\left(T_{1}-T_{c}, T_{c}\right)$. Using (1.9) we obtain the representation

$$
u\left(x, T_{1}\right)=\int \rho(\tau) U\left(T_{1}, \tau\right) U(\tau, 0) f(x) d \tau .
$$

Note that in general, if $(n-1) T_{c} \leq T_{1}<n T_{c}$ we would have to exploit this trick and the procedure which follows $n$ times to verify the theorem. We restrict ourselves to the simplest case $n=2$ for the sake of notational convenience.

Using the representation (1.7) we have

$$
\begin{aligned}
& u\left(x, T_{1}\right)=\int \rho(\tau) \int d \xi\left[\int_{|\omega|=1} \sum_{j=0}^{N} \sum_{\lambda=1}^{n} f_{j}\left(\phi^{\lambda}\left(\xi, \tau ; x, T_{1}, \omega\right)\right) a_{j, \lambda}\left(\xi, \tau ; T_{1}, \omega\right) d \omega\right. \\
& \left.+R_{N}\left(\xi, \tau ; x, T_{1}\right)\right] \\
& \int d y\left[\int_{\left|\omega^{\prime}\right|=1} \sum_{l=0}^{N} \sum_{\mu=1}^{n} f_{l}\left(\phi^{\mu}\left(y, 0 ; \xi, \tau, \omega^{\prime}\right)\right) a_{l, \mu}\left(y, 0 ; \xi, \tau, \omega^{\prime}\right) d \omega^{\prime}\right. \\
& \left.+R_{N}(y, 0 ; \xi, \tau)\right\rfloor f(y) d y
\end{aligned}
$$


and it suffices to show that the general term

$$
\int \rho(\tau) \int d \xi \int_{|\omega|=1} f_{j}\left(\phi^{\lambda}\right) a_{j, \lambda} d \omega \int d y \int_{\left|\omega^{\prime}\right|=1} f_{l}\left(\phi^{\mu}\right) a_{l, \mu} d \omega^{\prime} f(y) d \tau
$$

is $C^{\infty}$ except where the point $\left(x, T_{1}\right)$ can be traced back to the point $(y, 0)$ along a bicharacteristic through $(y, 0)$.

Let

$$
\Omega_{T_{1}}=\left\{\left(y, x^{\lambda}\left(T_{1} ; y, \omega, 0\right)\right):|\omega|=1, \lambda=1,2, \cdots, n\right\}
$$

where $x^{\lambda}\left(t ; y, \omega, t_{0}\right)$ is a solution to the Hamiltonian system

$$
\begin{array}{rlrl}
\frac{d}{d t} x^{\lambda} & =\tau_{\eta}^{\lambda}(x, t, \eta), & \frac{d^{\lambda}}{d t} \eta & =-\tau_{x}^{\lambda}(x, t, \eta) \\
x^{\lambda}\left(t_{0}\right)=y, & \eta^{\lambda}\left(t_{0}\right) & =\omega .
\end{array}
$$

For a given $r_{0}>0$ we can cover the complement of $\Omega_{T_{1}}$ with respect to $B_{K}(0) \times$ $B_{R}(0)$ by a finite collection of products of open balls $B_{r_{0}}\left(y_{j}\right) \times B_{r_{0}}\left(x_{j}\right)$. It suffices to consider a particular set in the covering, say $B_{r_{0}}(\tilde{y}) \times B_{r_{0}}(\tilde{x})$, and by introducing a partition of unity over $\operatorname{supp}(f)$ we can assume that $\operatorname{supp}(f) \subset B_{r_{0}}(\tilde{y})$. Set $M=\left\{r: 0 \leq \tau \leq T_{1}\right\} \times \overline{B_{r_{0}}(\tilde{x})} \times\{\xi:|\xi| \leq R\} \times \overline{B_{r_{0}}(\tilde{y})} \times\{\omega:|\omega|=1\} \times\left\{\omega^{\prime}:\left|\omega^{\prime}\right|=1\right\}$.

Claim. If $\bar{\nabla}$ denotes the gradient with respect to $\left(\xi, \tau, \omega, \omega^{\prime}\right)$, then on $M \cap$ $\left\{\phi^{\mu}=0\right\} \cap\left\{\phi^{\lambda}=0\right\}$,

$$
\bar{\nabla} \phi^{\mu}\left(y, 0 ; \xi, \tau, \omega^{\prime}\right) \# \bar{\nabla}_{\phi}^{\lambda}\left(\xi, \tau ; x, T_{1}, \omega\right) .
$$

Proof of Claim. Suppose not. Then there exists a point $p_{0}=\left(\tau_{0}, x_{0}, \xi_{0}, y_{0}\right.$, $\left.\omega_{0}, \omega_{0}^{\prime}\right)$ such that

(a) $\phi_{\omega^{\prime}}^{\mu}\left(y_{0}, 0 ; \xi_{0}, \tau_{0}, \omega_{0}^{\prime}\right)=0=\phi_{\omega}^{\lambda}\left(\xi_{0}, \tau_{0} ; x_{0}, T_{1}, \omega\right)$,

(b) $\left(\phi_{\tau}^{\mu}\left(p_{0}\right), \nabla_{\xi} \phi^{\mu}\left(p_{0}\right)\right) \|\left(\phi_{\tau}^{\lambda}\left(p_{0}\right), \nabla_{\xi} \phi^{\lambda}\left(p_{0}\right)\right)$.

Recall that $\phi^{\sigma}(z, s ; x, t, \omega)$ satisfies

$$
\phi_{t}^{\sigma}=\tau^{\sigma}\left(x, t, \nabla_{x} \phi^{\sigma}\right), \quad \phi^{\sigma}(z, s ; x, s, \omega)=(z-x) \cdot \omega .
$$

If $\eta$ represents any of the parameters in $\phi^{\sigma}$, that is, $z, s$ or $\omega$, set $\psi=\partial \phi^{\sigma} / \partial \eta$. An easy calculation shows that $\psi$ must be constant along the rays $x^{\sigma}(t ; z, \omega, s)$. In particular, since $\phi_{\omega^{\prime}}^{\mu}\left(y_{0}, 0 ; y_{0}, 0, \omega^{\prime}\right)=0$, it follows that $\phi_{\omega^{\prime}}^{\mu}\left(y_{0}, 0 ; x^{\mu}\left(\tau_{0} ; y_{0}, \omega_{0}^{\prime}, 0\right), \tau_{0}, \omega_{0}^{\prime}\right)=0$, and since $\phi^{\mu}\left(p_{0}\right)=0$, we see that $\xi_{0}=$ $x^{\mu}\left(\tau_{0} ; y_{0}, \omega_{0}^{\prime}, 0\right)$. Similarly, $x_{0}=x^{\lambda}\left(T_{1} ; \xi_{0}, \omega_{0}, \tau_{0}\right)$.

We also observe that $\partial \phi^{\lambda}\left(\xi_{0}, \tau ; \xi_{0}, \tau, \omega_{0}\right) / \partial \tau=0$, and since $\tau$ is a parameter in $\phi^{\lambda}$, we may conclude that $\partial \phi^{\lambda}\left(\xi_{0}, \tau_{0} ; x_{0}, T_{1}, \omega_{0}\right) / \partial \tau=0$. Since $T_{1}=\tau+t$, it follows that 


$$
\frac{\partial}{\partial \tau} \phi^{\lambda}\left(\xi_{0}, \tau_{0} ; x_{0}, T_{1}, \omega_{0}\right)=-\frac{\partial}{\partial t} \phi^{\lambda}\left(\xi_{0}, \tau_{0} ; x_{0}, T_{1}, \omega_{0}\right)=-\tau^{\lambda}\left(\xi_{0}, \tau_{0},-\omega_{0}\right) .
$$

Similarly, since $\xi$ is a parameter in $\phi^{\lambda}$, from the fact that $\nabla_{\xi} \phi^{\lambda}\left(\xi_{0}, \tau ; \xi_{0}, \tau, \omega_{0}\right)$ $=\omega_{0}$, it follows that $\nabla_{\xi} \phi^{\lambda}\left(p_{0}\right)=\omega_{0}$, and from (b) we see that $\nabla_{\xi} \phi^{\lambda}\left(p_{0}\right)=\alpha \omega_{0}$ for some $\alpha \neq 0$. Consequently $\phi_{\tau}^{\mu}\left(p_{0}\right)=\alpha \phi_{\tau}^{\lambda}\left(p_{0}\right)=-\alpha \tau^{\lambda}\left(\xi_{0}, \tau_{0},-\omega_{0}\right)$. But $\phi_{\tau}^{\mu}\left(p_{0}\right)=\tau^{\mu}\left(\xi_{0}, \tau_{0}, \nabla_{\xi} \phi^{\mu}\left(p_{0}\right)\right)=\tau^{\mu}\left(\xi_{0}, \tau_{0}, \alpha \omega_{0}\right)$ and therefore $-\alpha \tau^{\lambda}\left(\xi_{0}, \tau_{0},-\omega_{0}\right)$ $=\tau^{\mu}\left(\xi_{0}, \tau_{0}, \omega_{0}\right)$. Since the $\tau^{\prime}$ 's are positive homogeneous of degree 1 , we have that $\mp \tau^{\mu}\left(\xi_{0}, \tau_{0}, \pm \omega_{0}\right)=\tau^{\lambda}\left(\xi_{0}, \tau_{0},-\omega_{0}\right)$. As the equation is symmetric hyperbolic, the eigenvalues are necessarily all distinct, and since in general $\tau^{\mu}(\xi, \tau, \omega)=$ $-\tau^{\sigma}(\xi, \tau,-\omega)$ for a uniquely determined $\sigma$, it follows that $\tau^{\mu}\left(\xi, \tau_{0}, \omega\right)=$ ${ }^{\lambda}\left(\xi, \tau_{0}, \omega\right)$ for all $(\xi, \omega) \in\{\xi:|\xi| \leq R\} \times\{\omega:|\omega|=1\}$. Thus $\xi_{0}=x^{\mu}\left(\tau_{0} ; y_{0}, \omega_{0}^{\prime}, 0\right)$ and $x_{0}=x^{\mu}\left(T_{1} ; \xi_{0}, \omega_{0}, \tau_{0}\right)$ and therefore $\left(y_{0}, x_{0}\right) \in \Omega_{T_{1}}$, a contradiction.

Thus, on the set $M$ we have an open covering by neighborhoods $W$ of one of three types. Either

(1) $\phi^{\mu} \neq 0$ on $W$,

(2) $\phi^{\lambda} \neq 0$ on $W$, or

(3) $W$ is the product of spherical neighborhoods in $x$ and $y$ space with a neighborhood in $\left(\xi, \tau, \omega, \omega^{\prime}\right)$ space in which $\phi^{\lambda}$ and $\phi^{\mu}$ can be extended to a coordinate system; i.e., there exist smooth functions $\Phi_{\alpha}=\Phi_{\alpha}\left(\xi, \tau, \omega, \omega^{\prime}\right)$ such that $\left(\phi^{\lambda}, \phi^{\mu}, \Phi_{a}\right)$ can be introduced as a new coordinate system on $W$.

Since $M$ is compact, we may assume that this covering is finite and introduce a $C^{\infty}$ partition of unity on $M$ subordinate to this covering, which we use to evaluate (1.12). If the neighborhood is either of type (1) or type (2), it is clear that the kernel of the operator in (1.12) is $C^{\infty}$ except along the bicharacteristic rays. If the neighborhood $\Omega$ is of type (3), we first approximate the distributions $f_{j}$ and $f_{l}$ by $C^{\infty}$ functions $f_{j}^{\delta}$ and $f_{l}^{\delta}$, where $f_{j}^{\delta} \rightarrow f_{j}$ and $f_{l}^{\delta} \rightarrow f_{l}$ as $\delta \rightarrow 0$ in the weak topology of $\mathscr{I}^{\prime}(\Omega)$. Then we change the variables of integration in (1.11) to $\left(\phi^{\lambda}, \phi^{\mu}, \Phi_{a}\right)$ and by now letting $\delta \rightarrow 0$, we observe that the kernel of (1.12) converges to a $C^{\infty}$ function. Q.E.D.

II. Functions of the Gevrey type; Friedman's result. In what follows $\{H(q)\}_{q=1}^{\infty}$ will denote a monotone increasing sequence of positive real numbers satisfying the following four properties [2], [4]:

(i) $H(1) \geq 1$.

(ii) For all integers $p, q, 0 \leq p<q<\infty, \underset{p}{q}) H(p) H(q-p) \leq A H(q)$ for some constant $A \geq 1$; we define $H(0)=\Lambda$.

(iii) For all $q \geq 1$, there exist constants $\beta$ and $K_{0}$ such that $(H(q+1) / H(q))$ $\leq K_{0} \beta^{q}$.

(iv) $\sum_{q=1}^{\infty}(H(q) / H(q+1))<\infty$. 
Definition. Let $\Omega$ be an open set in $R^{k}$. $C(H(q), \Omega)$ will denote the set of all functions $f(x) \in C_{0}^{\infty}(\Omega)$ such that

$$
\sup _{x \in \Omega}\left|D^{\alpha} f(x)\right| \leq C K^{|a|} H(|\alpha|)
$$

where the constants $C$ and $K$ depend only on the function $f$ and we have used the multiple indice notation of $\mathrm{L}$. Schwartz.

Remark. It is easily shown that $C(H(q), \Omega)$ forms an algebra of functions.

The classic example of a sequence satisfying (i) to (iv) is given by $H(q)=$ $(q !)^{\mu}$, with $\mu>1$, and the corresponding functions $C\left((q !)^{\mu}, \Omega\right)$ are called the Gevrey functions of order $\mu$. Note that while only condition (iv) rules out the special case of $\mu=1$ (analytic functions), it is this property which is most crucial in what is to follow.

The following lemma was proved by S. Mandelbrojt [6].

Lemma 1. Let $H(q)$ be a monotone increasing sequence of positive real numbers satisfying condition (iv). Then there exists a nonnegative function $\rho_{\sigma}(x) \epsilon$ $C_{0}^{\infty}((-\sigma, \sigma))$ such that for all $n, \rho_{\sigma}^{(n)}( \pm \sigma)=0$ and

$$
\sup _{x \in[-\sigma, \sigma]}\left|D^{\alpha} \rho_{\sigma}(x)\right| \leq C(\sigma) K(\sigma)^{|\alpha|} H(|\alpha|) .
$$

Using a convolution construction, it follows that $C(H(q), \Omega)$ is dense in $\mathscr{D}(\Omega)$ and the following result is immediate.

Lemma 2 (C. Roumieu [7]). Let $\left\{\Omega_{j}\right\}$ by a countable covering of an open set $\Omega \subset R^{k}$ by the open sets $\Omega_{j}$. Then there exist functions $\beta_{i}(x) \in C\left(H(q), \Omega_{i}\right)$ such that for all $i, \beta_{i}(x) \geq 0$ and for each $x \in \Omega, \Sigma_{i} \beta_{i}(x)=1$.

Example. The classical example of a function in $C_{0}^{\infty}$, namely

$$
f(x)= \begin{cases}\exp \left(-\left(1-|x|^{2}\right)^{-1}\right), & |x|<1 \\ 0, & |x| \geq 1\end{cases}
$$

belongs to $C\left((q !)^{3},(-1,1)\right)$.

In this paper, A. Friedman [2] studied strictly hyperbolic systems of the form (1.1) using the following notation. Let $V$ be any open, bounded set of points in $(x, t)$ whose closure does not intersect the characteristic conoid emanating from $(y, 0)$. Set $V^{\sigma}=V \cap\{t: 0 \leq t \leq \sigma\}$ and denote by $W_{K}^{\sigma}$ the cylinder $\{(x, t): 0 \leq t \leq \sigma,|x| \leq K\}$. Let $\epsilon_{1}>0$ be the time at which the first caustic appears and consider the infinite slab $0 \leq t \leq \epsilon_{1}$. Working within this domain, if we are given $R_{0}>0$, we can select $R, 0<R<R_{0}$, and $\epsilon>0$ so that the domain of dependence of $W_{R}^{\epsilon}$ is contained within $W_{R_{0}}^{\epsilon}$ and $V^{\epsilon} \subset W_{R}^{\epsilon}$. With this construction in mind, we have the following result. 
Theorem 2. If the coefficient matrices $A^{j}(x, t), B(x, t)$ belong to $C\left(H(q), W_{R_{0}}^{\epsilon}\right)$, then the Riemann function $\underset{\sim}{R}(y, 0 ; x, t)$ belongs to $C\left(\tilde{H}(q), V^{\epsilon}\right)$ for some $\epsilon=\epsilon\left(R, R_{0}\right)$ sufficiently small and $\tilde{H}(q) \leq H(3 q) /(q !)$.

Remark. This result was proved assuming only properties (i) and (ii) for the sequence $H(q)$. In particular, the analytic case was handled and it was shown that for this special case, $\widetilde{H}(q)=H(q)=q$ !

The method of proof involves obtaining estimates on each of the terms in the expansion (1.7) by using a series of preliminary results on the algebra of functions $C(H(q), \Omega)$. The most difficult estimate, and the one which introduced the new sequence $\tilde{H}(q)$ involved the terms $a_{j, \lambda}(y, s ; x, t, \omega)$.

Implicit in the statement of Theorem 2 was the requirement that $\epsilon<\epsilon_{1}$. In view of Theorem 1, however, this restriction seems artificial. In particular, if we knew that $R_{0}$ was sufficiently large so that the domain of dependence of $W_{R}^{\epsilon} 0$ is contained within $W_{R_{0}}^{\epsilon_{0}}$ and that $V^{\epsilon_{0}} \subset W_{R}^{\epsilon_{0}}$, we have the more general result.

Theorem 3. If the coefficient matrices $A^{j}(x, t), \underset{\sim}{B(x, t)}$ belong to $\underset{\sim}{C}\left(H(q), W_{R_{0}}^{\epsilon_{0}}\right)$, then the Riemann function $R(y, 0 ; x, t)$ belongs to $C\left(\tilde{H}(q), v^{\epsilon}\right)$ with $\tilde{H}(q) \leq$ $H(3 q) / q$ !.

Proof. It is sufficient to extend the result beyond caustics using the method of Theorem 1 and the previously obtained estimates of Friedman in domains where no caustics appear. In particular, since we already know that the right hand side of the expansion (1.11) is $C^{\infty}$ except along the bicharacteristics through $(y, 0)$, and we have estimates on each of the terms which appear, by choosing $\rho(\tau) \epsilon$ $C\left(\tilde{H}(q),\left(0, T_{c}\right)\right)$ (Lemma 1$)$, it is clear from the algebraic properties of $C(\tilde{H}(q), \Omega)$ that with the possible exception of terms like (1.12), all remaining terms belong to $C(\tilde{H}(q), \Omega)$. We turn, therefore, to the terms (1.12). As before, we evaluate this expression by expanding with a finite partition of unity of $M$, which may be chosen in $C(\tilde{H}(q), W)$ (Lemma 2). If the neighborhood is of either type (1) or type (2), the result is immediate. For neighborhoods of type (3), we approximate the distributions $f_{j}\left(f_{l}\right)$ by functions in $C(\tilde{H}(q), W)$, which is known to be dense in $\mathscr{D}(\Omega)$. By Friedman's analog of the implicit function theorem for functions in $C(\tilde{H}(q), \Omega)$, the Jacobian $\left|\partial\left(\xi, \tau, \omega, \omega^{\prime}\right) / \partial\left(\phi^{\lambda}, \phi^{\mu}, \Phi_{\alpha}\right)\right|$ is in $C(\tilde{H}(q), W)$, and proceeding as in Theorem 1, the proof is complete. Q.E.D.

\section{BIBLIOGRAPHY}

1. R. Courant and P. D. Lax, The propagation of discontinuities in wave motion, Proc. Nat. Acad. Sci. U. S. A. 42 (1956), 872-876. MR 18, 399.

2. A. Friedman, Regularity of fundamental solutions of hyperbolic equations, Arch. Rational Mech. Anal. 11 (1962), 62-96. MR $26 \# 1624$. 
3. P. D. Lax, (a) Asymptotic solutions of oscillatory initial value problems, Duke Math. J. 24 (1957), 627-646. MR 20 \#4096. (b) Lectures on hyperbolic differential equations, Stanford University, Stanford, Calif., 1963.

4. J. L. Lions and E. Magenes, Problèmes aux limites non homogènes et applications. Vol. 3, Dunod, Paris, 1970.

5. D. Ludwig, Exact and asymptotic solutions of the Cauchy problem, Atomic Energy Commission Research and Development Report NYO 2545.

6. S. Mandelbrojt, Séries adhérentes régularisation des suites, applications, GauthierVillars, Paris, 1952. MR 14, 542.

7. C. Roumieu, Sur quelques extensions de la notion de distribution, Ann. Sci. École Norm. Sup. (3) 77 (1960), 41-121. MR 22 \#12377.

DEPARTMENT OF MATHEMATICS, UNIVERSITY OF NOTRE DAME, NOTRE DAME, INDIANA 46556 